\title{
HUMAN GUT MICROBIOTA AND ITS EFFECTS ON HUMAN HEALTH IN NORMAL AND PATHOLOGICAL CONDITIONS
}

\author{
Univ. Assist. Anca Magdalena Munteanu ${ }^{1,2}$, MD, PhD, Raluca Cursaru ${ }^{2}$, MD, Loreta Guja ${ }^{2}$, MD, \\ Simona Carniciu ${ }^{5}, \mathrm{MD}, \mathrm{PhD}$, Univ. Assist. Alina Maria Borcan ${ }^{2,4}, \mathrm{MD}, \mathrm{PhD}$, \\ Lecturer Dana Popescu-Spineni ${ }^{2,3}$, MD, PhD \\ ${ }^{\prime}$ National Institute of Public Health, Bucharest \\ ${ }^{2}$ University of Medicine and Pharmacy "Carol Davila", Bucharest \\ ${ }^{3}$ Institute of Anthropology "Fr. I. Rainer" of the Romanian Academy, Bucharest \\ ${ }^{4}$ National Institute of Infectious Diseases "Prof. Dr. Matei Bals", Bucharest \\ ${ }^{5}$ Center of Research in Diabetes and Nutrition Disorders "Corposana", Bucharest
}

\begin{abstract}
The medical research of the last 1-2 decades allows us to look at the human gut microbiota and microbiome as to a structure that can promote health and sometimes initiate disease. It works like an endocrine organ: releasing specific metabolites, using environmental inputs, e.g. diet, or acting through its structural compounds, that signal human host receptors, to finally contributing to the pathogenesis of several gastrointestinal and non-gastrointestinal diseases. The same commensal microbes were found as shapers of the human host response to drugs (cardiovascular, oncology etc.). New technologies played an important role in these achievements, facilitating analysis of the genetic and metabolic profile of this microbial community. Once the inputs, the pathways and a lot of human host receptors were highlighted, the scientists were encouraged to go further into research, in order to develop new pathogenic therapies, targeting the human gut flora. Dual therapies, envolving these "friend microbes", are another actual research subjects. This review gives an update on the current knowledge in the area of microbiota disbalances under environmental factors, the contribution of gut microbiota and microbiome to the pathogenesis of obesity, obesity associated metabolic disorders and cardiovascular disease, as well as new perspectives in preventing and treating these diseases, with high prevalence in contemporary, economically developed societies. It brings the latest and most relevant evidences relating to: probiotics, prebiotics, polyphenols and faecal microbiota transplantation, dietary nutrient manipulation, microbial as well as human host enzyme manipulation, shaping human responses to currently used drugs, manipulating the gut microbiome by horizontal gene transfer.
\end{abstract}

Keywords: environment, dietary nutrients, gut microbiota, microbiome, probiotics, prebiotics, polyphenols, antibiotics, faecal microbiota transplantation, dietary nutrient manipulation, enzyme manipulation, dual therapies

\section{INTRODUCTION}

The gut microbiota - the largest microorganism community living symbiotic inside the human body - currently focuses more and more research, showing that these microbes are not temporal passengers in the host (1). The functions of gut microbiota were noted in the latest sequencing technologies and metabolite profiling. It promotes a good health, the initiation or maintenance of different diseases. From here, the perspective of new treatments of the diseases, targeting microbiota, manipulating its metabolome emerges; the scientists of our present days aim to integrate an individual's microbiota into some form of personalised healthcare and, by better understanding its role, treat an individual's diseases more efficiently and in a more targeted fashion. Therefore, this review tries to gather recent knowledge about human gut microbiota, the gut microbiome as endocrine organ and the relationship with several chronic diseases prevalent in the modern society, and, last but not least, new possible interventions to prevent or treat such diseases, targeting human gut microbiota and microbiome (1).

Correspondence address:

Simona Carniciu, MD, PhD, Center of Research in Diabetes and Nutrition Disorders "Corposana", Bucharest E-mail: cassimona@gmail.com 


\section{Human gut microbiota}

Usually fetuses are sterile in the womb, and until the age of 4 years old a lot of microbes colonize the gut and this remains constant until the 7 th decade. The microbes come from mother, at birth, and from the environment, the first years after $(2,3)$. The mature gut flora contains 500 up to 1,000 different bacterial species (4). The number of bacteria progressively increases along the small bowel from $\sim 10^{4}$ in the jejunum to $10^{7}$ colony-forming units per gram of luminal content at the ileal end, with a predominance of gram-negative aerobes. In the colon, the bacterial count reaches around $10^{12}$ colonyforming units per gram with a predominance of anaerobes (4).

Most of the bacteria are the gram-positive Firmicutes and Actinobacteria and the gram-negative Bacteroidetes. The Firmicutes is the largest bacterial phylum (division), including Lactobacillus, Mycoplasma, Bacillus and Clostridium species (4). The human gut flora is important for maintaining a healthy state and promoting some diseases. We'll discuss later on obesity and other associated metabolic disorders that are associated with dysbiosis (disturbances in microbiota composition) (5). The composition of microbiota is similar only to related people, in that time the functions contributed by these species appear to be found in everybody's gastrointestinal tract. Although different species meet common functions, there might be a difference, consisting in the effectiveness of a function (1). For example, while the ability to synthesize short chain fatty acids (SCFAs) is found in all individuals, their amounts can vary (6). Why in unrelated people gut microbiota has such a large diversity? Because of the great influence the environment appears to have on it. Nutritional factors such drinking nonchlorinated water, eating whole grain, breastfeed, physical activity and perform natural births play an important role in maintaining a „healthy“ microbiome (7). In contrast to „healthy“ microbiome is ,westernized" microbiome, which is characterized by excessive use of antibiotics, consumption of highly processed foods, increased rates of caesarean sections and other factors (8).

In our lifestyle, four interventions are important for boosting good bacterial populations: eating more traditionally fermented foods and fermentable substrates, avoiding antibiotics and ,getting dirty" once in a while. More precisely:

- Instead of the modern low-fat milk we should aim for products such as dirty fermented veggies, grass-fed Greek yoghurt and thousand of years old kefir grains (8).
- We should eat more fermentable substrates/ prebiotics such as inulin-type fructans (leeks, onions and Jerusalem artichoke) and resistant starches (green bananas, legumes and potatoes, among other foods). As cooking breaks down a lot of the resistant starch found in food, cooling legumes and potatoes for about 36 hours after heating is a good approach. This increases the resistant starch content through a process called starch retrogradation (8-10).

In non-industrialized societies people consume much higher fibre than the low norms of Western societies (11). A recent study which analysed the effects of 55 grams of daily fibre $(11,12)$.

- Besides other known factors that participate in the destruction of the ecosystem, the use of antibiotics, in particular the broad spectrum, also promotes the destruction of the gut microbiome (8).

- A lack of microbial exposures increases the risk of developing diseases (8).

A reason for decrease the incidence of inflammatory diseases seems to be microbiome of hunter-gatherers and non-westernized populations which is different from westerners' microbiome $(8,13,14)$.

\section{Human gut microbiome in the pathogenesis of certain chronic diseases, prevalent in contemporary societies}

Gut microbiome in obesity and other obesity associated metabolic disorders

In the recent research gut microbiota appears to be involved in the development of metabolic diseases. Gut microbiota is seen as a separate organ who play an important role in maintaining homeostasis and stimulating immunity. The external factors can lead to the development of metabolic disorders induced by changes in the gut microbiome thereby affecting the relationship between the host and the gut bacteria $(15,16)$. The researchers found that the gut microbiota promotes insulin resistance, fat deposition and low grade inflammation and can involve in the onset of metabolic diseases and obesity (Fig. 1).

For people following a Western style diet the carbohydrate fermentation occurs in the colon, more specifically in the proximal colon. After this process results short-chain fatty acids (SCFAs) that serve as energy substrate for gut microbiome and host. Moreover, $10 \%$ of our daily energy requirements are coming from colonic fermentation $(1,17)$. Some SCFAs such as butyrate and propionate seem 
to be involved in the immune function and the proper functioning of the gut and acetate acts as a substrate for lipogenesis and gluconeogenesis $(1,17)$. These metabolites have been identified in the resolution of inflammation, regulating the inflammatory output of adipose tissue and immune function $(1,18)$. Thus, we can appreciate the important role of carbohydrate fermentation in the gut, this representing the scientific starting point for improving intestinal health, and approaching the relationships between gut microbiome and liver, brain and even skin $(1,16)$.

Obesity is the result of imbalance between intake and expenditure. It was observed that these individuals with obesity and its associated metabolic diseases present a lower production of butyrate and reduced bacterial diversity most likely due to inflammation. Differences in gut microbial ecology might be an important mediator and it represents a biomarker for predicting metabolic dysfunction in the future. The imbalance between some intestinal bacteria (increase of Firmicutes and decrease in Bacteroidetes) was associated with the microbiota of obese mice. This thing was also observed in human: weight loss of obese individuals was associated with an increase of Bacteroidetes $(1,19,20)$.

In another study, which analyzed a bimodal distribution of microbial gene richness in obese individuals was noted that individuals with High Gene Count had a higher prevalence of $F$. prausnitzii (an- ti-inflammatory species) and an increased production of butyrate. In contrast, the individuals with Low Gene Count (LGC) had a higher prevalence of Bacteroides spp (proinflammatory) (1). Another study found that a diet-induced weight-loss intervention significantly increased LGC individuals and the metabolic status of these individuals was improved $(1,21)$. Based on these studies, we can say that the gut microbiome components are permanently influenced by diet.

Besides the known etiopathogenic factors involved in the increasing prevalence of obesity and the fact that some individuals seem more susceptible to diet-induced weight gain, hyperglycaemia should also be taken into account. The pathophysiological processes driving this relationship have not been completely elucidated $(1,22-24)$.

In lean people the gut microbiota is dominated by Bacteroidetes. It was observed that a carbohydrate-restricted or fat-restricted diet for 52 weeks leads to an increase of Bacteroidetes species in obese human (25) and this thing was correlated with weight loss $(20,26)$. In contrast, in the obese individuals predominate Firmicutes species in the distal gut associated with a pro-inflammatory state. The use of probiotics (Bifidobacterium, Lactobacillus) promotes correcting dysbiosis by increasing production of SCFAs $(8,27,28)$. Some researchers found that there is no significant difference between supplementation with probiotics and placebo

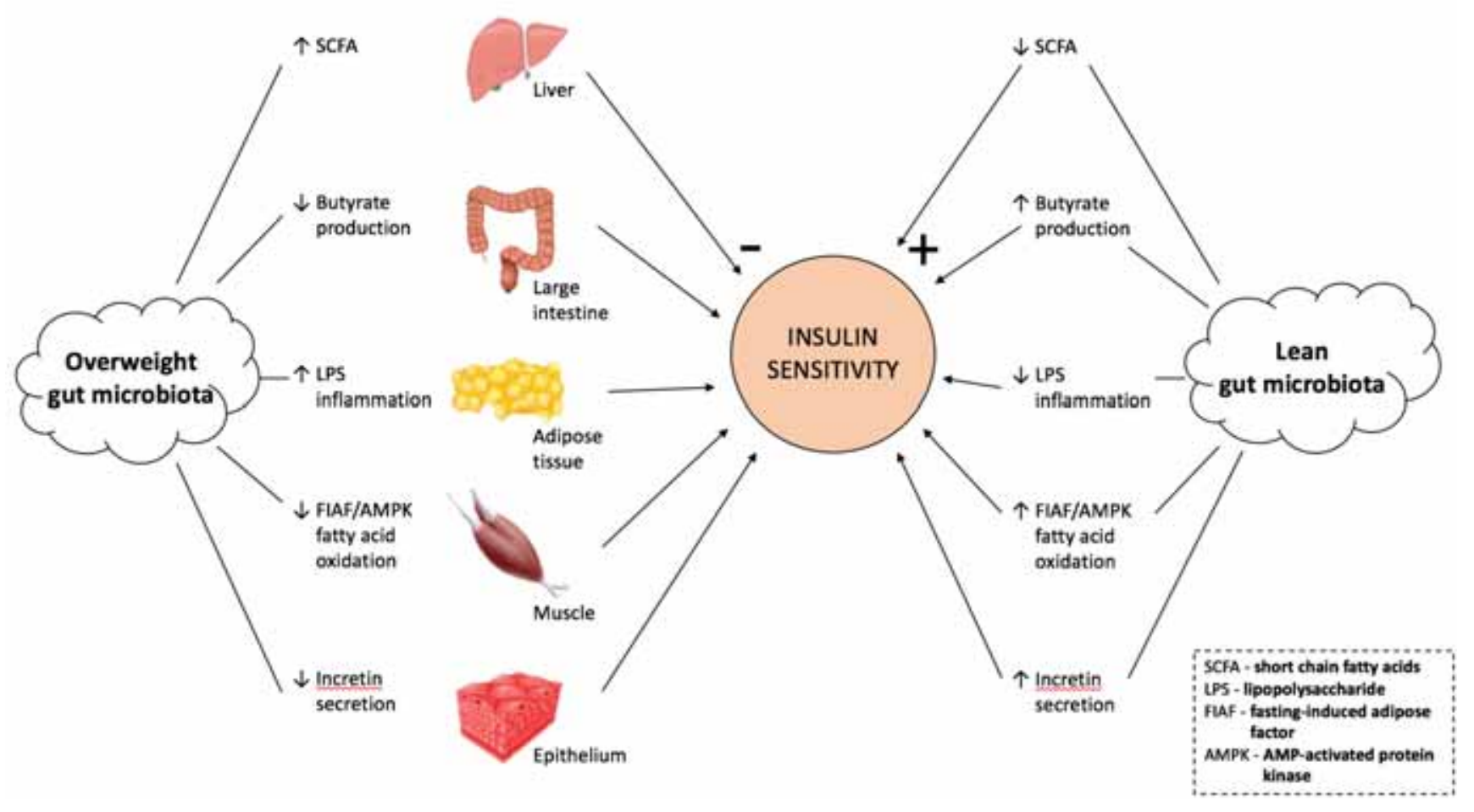

FIGURE 1. Metabolic activities of gut microbiota, lean vs. obese people 
on weight reduction. On the other hand some studies showed the contrary (28).

\section{Perspective interventions in obesity}

The analysis of intestinal bacteria can be a starting point for developing new therapies for reduce the prevalence of obesity and its related diseases.

Faecal microbiota transplantation (FMT) represents a method by which a faecal sample is transferred from a healthy person into a patient through nasogastric tube, nasoduodenal tube, rectal enema or the biopsy channel of a colonoscope $(1,29)$. One study that examined a group of men with metabolic syndrome who have undergone a faecal transplantation with stools from healthy lean individuals demonstrated that these pacients had improved insulin sensitivity and triglycerides comparared with men who underwent a transplant of their own stool (30).

Food supplements such probiotics are a real benefit to the human body by improving the intestinal microbiota and also impact the balance of the colonic microbiota. It was observed that in mice fed a high-fat diet, Bifidobacterium spp lead to improve glucose homeostasis, reduce weight gain and fat mass (30).

Prebiotics can be found in the common food, especially in cereals and vegetables. The most common prebiotic are fructosyl-oligosaccharides (FOS) such inulin, galactosyl-oligosaccharides (GOS) and they are transformed by the gut microbiota into SCFAs (30-32). Although the results of the studies on the effect of prebiotics in human are still contradictory and require further researches, it has been demonstrated that prebiotics regulate the fecal microbiota and reduce markers of metabolic syndrome $(30,33,34)$.

Polyphenols are a class of plant secondary metabolites. They enhance the beneficial, anti-inflammatory bifidobacteria and lactobacilli (1). From here the perspective of functional foods to regulate gut microbiome and contribute to the health of the intestinal microbiota and its host.

\section{Gut microbiome and cardiovascular disease (CVD)}

The gut microbiota is considered to be an endocrine organ due its functions and its hormone-like signals, allowing new perspectives in the prevention and treatment of the atherosclerosis and other cardiovascular diseases (CVD) $(15,35)$. CVD is a leading cause of death in many industrialized countries and, of course, the interest in its prevention and treatment is an increased one. The main pre- vention consists of controlling the environmental pathogenesis and its main exposure, which is the food that we eat. Our digestive system contains trillions of bacteria that made up the gut microbiota, a reliable filter between a host and the environment, and its exact role in our immune function and health is still a blank area $(13,35)$.

The relationship between the food, gut microbiota, liver and CVD is rather simple: some food rich in lecithin, carnitine and choline and containing trimethylamine (TMA) are metabolized by the microbe specific enzymes when meet the gut microbiota and release the TMA into the gut lumen. The TMA is transported to the human liver, where it is transformed in trimethylamine-N-oxide (TMAO) (36-41), enters in circulation and then it is excreted by the kidney. In studies, TMAO seems to be associated with increasing cholesterol, atherosclerosis development, increase risk of myocardial infarction (MI), stroke (35). The role of microbiota was proved in studies: the ingestion of egg-yolk (source of phosphatidilcholine) or of red meat (source of L-carnitine) with plasma and urine levels of TMA and TMAO, in the presence or absence of an antibiotic cocktail (encharged to manipulate the gut microbiota) (42-44). Metabolism-dependent and independent effects of the gut microbial endocrine organ converge to modulate risk of developing atherosclerotic CVD, MI, stroke and death (Fig. 2). Supplementing diet with TMAO and choline determines increased expression of scavenger receptors (CD36 and SR-A1) on macrophages and leads to foam cell formation. Use a TMAO receptor system would be a major advance and provide an attractive therapeutic target for atherosclerotic CVD (45).

In a study with more than 4,000 subjects who underwent elective coronary angiography demonstrated that increased levels of TMAO has been associated with major adverse cardiac events (42). The CVD predictive power of TMAO plasma level functions both in healthy people and in people caring chronic renal disease (46). TMAO plasma level is a much more sensitive prognostic indicator than cardio-renal indices (glomerular filtration rate and B-type natriuretic peptide) and C-reactive protein - a marker of systemic inflammation (47). TMAO can lead to impaired glucose tolerance and promotes adipose tissue inflammation in mice fed a high-fat diet (48).

\section{SCFAs}

The carbohydrates like hemicelluloses, pectins, galactose and others are undergoing intestinal fermentation process due to anaerobic bacteria. The 

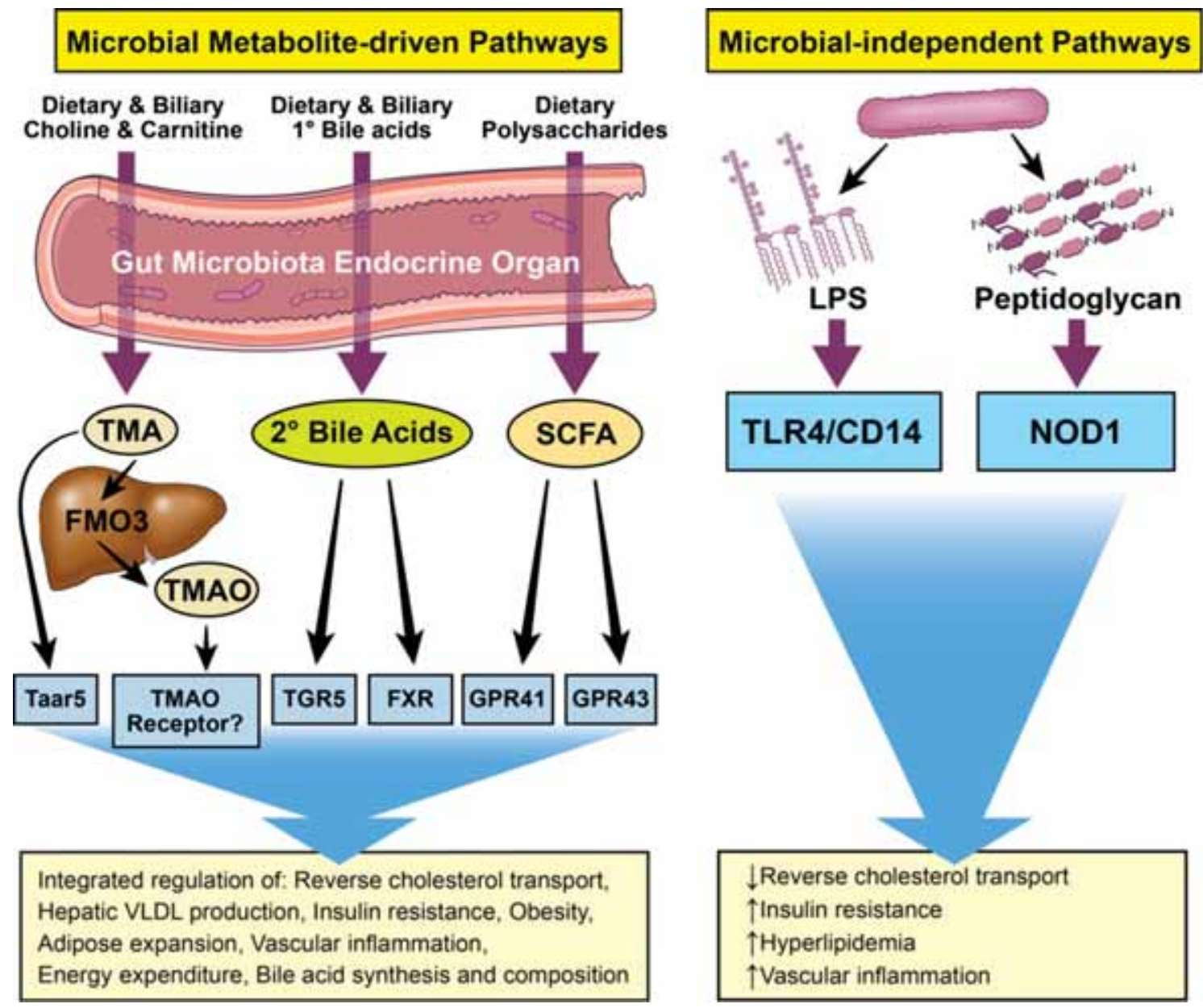

FIGURE 2. Host Receptor Systems for Sensing Bacterial Products Relevant to Cardiovascular Disease (45)

metabolites result from this process are in fact short chain fatty acids (SCFAs), including acetic acid, butyric acid, propionic acid and valeric acid. In the host SCFAs increase energy expenditure regulating energy metabolism, improve insulin sensitivity and regulate metabolic responses through action on the central nervous system (45).

\section{Bacteria-modified bile acids}

Into the body, one of the role of bile acids is to solubilize dietary lipids. In the recent researches the bile acids seem to be involved in the insulin sensitivity, energy expenditure and cholesterol synthesis (45). A small amount of bile acids together with anaerobic bacteria forms microbe-dependent secondary bile acids in the large intestine. Some of them get into the circulation and promote the increase of brown adipose tissue, insulin sensitivity and energy expenditure. So, the relationship between microbiota and bile acids could explain cardiometabolic pathways (45, 49, 50-55).

Lipopolysaccharide (LPS) and peptidoglycans are products of bacterial cell wall and a lower level of these is associated with insulin resistance, inflammation and hyperlipidemia. Low levels of gut-derived bacteria appear in type 2 diabetes, obesity, atherosclerosis (45).

\section{Prevention and treatment perspectives}

All the research summarized above leads to the pathway involving diet, gut microbiota and its composition pattern, TMA and TMAO. They represent a new paradigm for researchers. The release of a pathway allows potential interventions suggested for reducing TMAO generation (Fig. 3).

Modifying dietary substrate. Choline is essential for humans and it cannot be synthesized in our body. It is abundant in egg yolk, meats, liver, fish, high-fat dairy products and some nuts (56). Severe choline deficiency affects the nervous system (57). The gut microbiota is exposed to choline not only by diet, but by the endogenous bile too. The most feasible intervention might be using choline absorbents (58-60).

L-carnitine is produced by the body from dietary lysine, the single most abundant amino acid in 
plant and animal proteins $(38,61-62)$. As to the L-carnitine, the feasible intervention is eliminating red meat systematic intake. It is important to continue to identify dietary amines which are implicated in metabolic pathways.

Manipulating microbial metabolism. Two enzymes (C-N bond lyases, choline-specific and carnitine-specific) were found out until present, including the genes that control their synthesis, in genomic research of choline-degrading bacterium Desulfovibrio desulfuricans $(63,64)$ and of carnitine-degrading bacterium Acinetobacter baumannii (65).

From here comes another two therapeutic strategies to limit TMAO production: one consisting of targeting these enzymes (66) and other, much used in clinical practice, is modulating the microbial community through ingestion of a probiotic or prebiotic.
The prebiotic ingestion of dietary fructans (fruits and vegetables) has a beneficial effect on Bifidobacteria. In this way dietary-fructan improves insulin resistance. In addition to prebiotic approaches, several recent studies emphasize the potential utility of probiotics in modulating host metabolism and disease (45). The probiotic administration of a genetically-modified strain of bacteria has shown to protect mice from obesity and its associated disorders.

The above examples support the utility of prebiotic and probiotic in clinical practice and their potential role in cardiometabolic diseases but further research are required for a fully elucidated mechanisms of action of them.

Effects of hepatic FMOs on host metabolism

Human enzymes like FMO1, FMO2 and FMO3 form TMAO. FMO3 seems to be more investigated than other FMOs (44). Some studies have found

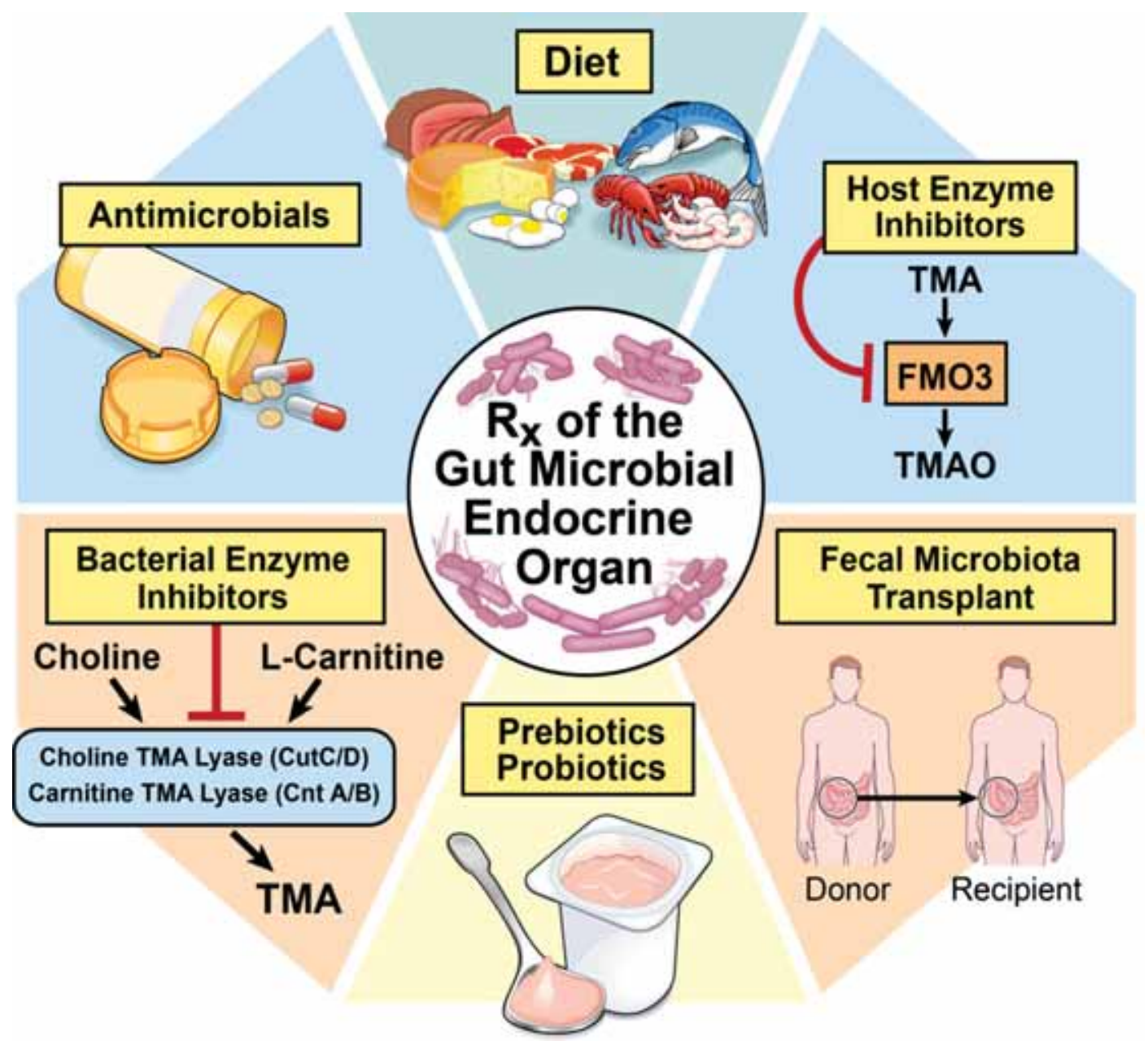

FIGURE 3. Strategies to Target the Gut Microbial Endocrine Organ for Improving Cardiovascular Disease

Source: Brown JM, Hazen SL. The Gut Microbial Endocrine Organ: Bacterially-Derived Signals Driving Cardiometabolic Diseases. Annual review of medicine. 2015; 66:343-359. doi:10.1146/annurev-

med-060513-093205 (45) 
that diet and gut microbiota have a greater effect on TMAO than host genetic (3).

Consequently, the therapeutic strategy on host hepatic enzyme-dependent might be the reduction of TMAO production due to inhibition of the FMOs (3). But this process can lead to a fish odor syndrome or trimethylaminuria $(2,67)$. Another therapeutic key is to block the ability of circulating TMAO. Use of the absorbent agents can represent a therapeutic option. Another therapeutic strategy could be to intercept TMAO at the molecular receptor (45).

The gut microbiota are capable to metabolize xenobiotics, thereby influencing health condition. For example digoxin can be inactivated by Actinobacteria $(45,68)$.

It is difficult to believe that two microbiological concepts will change the design of cardiovascular drugs: on one side, it will be critical to isolate by culture gut microbes in order to develop the probiotic class of therapeutics; on the other side, it is critical to emphasize the technique of horizontal gene transfer (HTG) $(45,69)$. HGT consists in the transfer of genes between organisms in a manner that does not rely on reproduction (45). Although the intestinal microbes of human have a higher rate of HGT, it was shown that after transferring of a marine bacterial gene to a human resident symbiont has resulted in the digestion of seaweed polysaccharides $(45,70)$.

\section{CONCLUSIONS}

In biomedical research of the last decades, the analysis of human gut microbiota, proving its contributions to human host physiology, to several disease pathogenesis and to human host response to drugs represents a top issue. Nowadays, research highlights the endocrine role played by the gut microbiota within the human host, through both dependent and independent mechanisms.
Our diet, the largest environmental exposure, provides inputs for this huge microbial endocrine organ; its own metabolic pathways allow turning the nutrient inputs into unique metabolits (short chain fatty acids, secondary biliary acids, trimethylamine and its oxidized form TMAO, host dependent) that have been shown to signal human host receptors, finally considered in the pathogenesis of diseases such as obesity, obesity associated metabolic disorders, diabetes, atherogenesis. As well, microbial metabolism-independent signals have regulatory roles in the development of cardiovascular disease.

The next step from here is to develop and consolidate new pathogenic therapies of such chronic metabolic diseases, targeting gut microbiota. As we look at the therapeutic strategies, its up to date to consider intervention at different levels of the pathogenic pathway, such as: absorbing involved dietary nutrients (choline, L-carnitine) to diminish the inputs, modulating the microbiota by probiotic and prebiotic supplementation, or by faeces microbiota transplantation, inactivating microbial enzymes responsible of TMA synthesis or using absorbent agents to bind and eliminate TMA and TMAO from the intestine.

Not of less interest is the research to improve human response to drugs, targeting the same gut microbiota. Both directions, that of shaping human response to traditional drugs and that of using new interventions, by manipulating gut microbiota and microbiome, still need more research.

There is still great need of analytical approaches that integrate data from food diary, data about lifestyle (stress, inactivity), antibiotic use to understand the roles of intestinal bacteria in human obesity. This knowledge would help to optimize therapeutic strategies to reshape the gut microbial ecology, as well as to improve the stratification of populations at risk of developing metabolic diseases and would offer novel perspectives for a personalized health care.

\section{REFERENCES}

1. Ayesh R., Mitchell S.C., Zhang A., Smith R.L. The fish odour syndrome: biochemical, familial, and clinical aspects. BMJ. 1993; 307(6905):655-657.

2. Xu J., Gordon J.I., Inaugural article: honor thy symbionts. Proc Natl Acad Sci USA.2003,100:10452-10459

3. Zoetendal E.G., Akkermans A.D., de Vos W.M. The host genotype affects the bacterial community in the human gastrointestinal tract. Microb Ecol Health Dis, 2001,13:129-134

4. Zoetendal E.G., Vaughan E.E., de Vos W.M. A microbial world within us. MolMicrobiol 2006; 59:1639-1650

5. Hobden Mark R., Laetitia Guerin-Deremaux, lan Rowland, Glenn R. Gibson, Orla B. Kennedy. Potential anti-obesogenic properties of non-digestible carbohydrates: specific focus on resistant dextrin. Proceedings of The Nutrition Society, 2015.

6. Flint H.J., Scott K.P., Louis P. et al. The role of the gut microbiota in nutrition and health. Nature Rev Gastroenterol Hepatol. 2012, 9:577-89.

7. Contreras B., 4 Keys To Better Gut Health And Improved Fitness, $\mathrm{https://bretcontreras.com/4-keys-to-better-gut-health-and-improved-}$ fitness/; 
8. Schneiderhan Jill Master-Hunter, Tara L. Targeting gut flora to treat and prevent disease. Journal of Family Practice, Jan 2016 Issue.

9. Selhub E.M., Logan A.C., Bested A.C. Fermented foods, microbiota, and mental health: ancient practice meets nutritional psychiatry. Journal of Physiological Anthropology. 2014,33(1):2.

10. Slavin J. Fiber and Prebiotics: Mechanisms and Health Benefits. Nutrients. 2013;5(4):1417-1435.

11. Deehan and Walter. The Fiber Gap and the Diasappearing Gut Microbiome: Implications for Human Nutrition. Trends in Endocrinology \& Metabolism, 2016 DOI:10.1016/j.tem.2016.03.001

12. Erica D. Sonnenburg, Samuel A. Smits, Mikhail Tikhonov, Steven K. Higginbottom, Ned S. Wingreen, Justin L. Sonnenburg. Dietinduced extinctions in the gut microbiota compound over generations. Nature, 2016; 529 (7585): 212 DOI: 10.1038/ nature 16504

13. Tito R.Y., Knights D., Metcalf J., Obregon-Tito A.J., Cleeland L., Najar F. et al. (2012) Insights from Characterizing Extinct Human Gut Microbiomes. PLoS ONE 7(12): e51146. doi:10.1371/journal. pone. 0051146

14. Jeff Leach February 20, 2013 Human Food Project, http:// humanfoodproject.com/slumdog-microbiome-more-diverse/;

15. Marchesi J.R., Adams D.H., Fava F. et al. plus BOLD The gut microbiota and host health: a new clinical frontier, Gut 2016; 65:330-339

16. Clarke G., Stilling R.M., Kennedy P.J., Stanton C., Cryan J.F., Dinan T.G. Minireview. Gut microbiota: the neglected endocrine organ. Mol Endocrinol. 2014; 28:1221-38. doi:10.1210/me.2014-1108.

17. Macfarlane G.T., Macfarlane S., Fermentation in the human large intestine: its physiologic consequences and the potential contribution of prebiotics. J Clin Gastroenterol 2011; 45(Suppl):S120-7. doi:10.1097/MCG.0b013e31822fecfe

18. Arpaia N., Campbell C., Fan X. et al. Metabolites produced by commensal bacteria promote peripheral regulatory T-cell generation. Nature 2013; 504:451-5. doi:10.1038/nature12726;

19. Backhed F., Turnbaugh P. et al. Obesity alters gut microbial ecology. Proc Natl Acad Sci USA 2005; 102:11070-5. doi:10.1073/ pnas.0504978102;

20. Ley R.E., Turnbaugh P.J., Klein S. et al. Microbial ecology: human gut microbes associated with obesity. Nature 2006; 444:10223. doi:10.1038/4441022a;

21. Cotillard A., Kennedy S.P., Kong L.C. et al. Dietary intervention impact on gut microbial gene richness. Nature 2013; 500:5858. doi:10.1038/nature 12480

22. Hill J.O., Peters J.C. (1998) Environmental contributions to the obesity epidemic. Science 280:1371-1374

23. Christakis N.A., Fowler J.H. (2007) The spread of obesity in a large social network over 32 years. N Engl J Med 357:370-379

24. Turnbaugh P.J., Hamady M., Yatsunenko T. et al. (2009) A core gut microbiome in obese and lean twins. Nature 457:480-484

25. Vrieze A., Holleman F., Zoetendal E.G. et al., The environment within: how gut microbiota may influence metabolism and body composition, in Diabetologia (2010) 53: 606. doi:10.1007/s00125-0101662-7, https://link.springer.com/article/10.1007/s00125-010-1662-7

26. Nadal I., Santacruz A., Marcos A. et al. (2008), Shifts in clostridia, bacteroides and immunoglobulin-coating fecal bacteria associated with weight loss in obese adolescents. Int J Obes (Lond) 33:758-767

27. Devaraj S., Hemarajata P, Versalovic J. The human gut microbiome and body metabolism: implications for obesity and diabetes. Clin Chem. 2013; 59:617-628.

28. Park S., Bae J.H. Probiotics for weight loss: a systematic review and meta-analysis. Nutr Res. 2015; 35:566-575.

29. NICE (National Institute for Health and Care Excellence). Faecal microbiota transplant for recurrent Clostridium difficile infection, NICE Interventional Procedure Guidance 2014:485.

30. Weilin Wang, Shaoyan Xu, Zhigang Ren, Jianwen Jiang, Shusen Zheng. Gut microbiota and allogeneic transplantation. Journal of translational Medicine, 2015. DOI: 10.1186/s12967-015-0640-8.

31. Delzenne N.M., Kok N. Effects of fructans-type prebiotics on lipid metabolism. Am J Clin Nutr. 2001; 73(2 Suppl):456S-8S.
32. Daddaoua A., Puerta V., Requena P., Martinez-Ferez A., Guadix E., de Medina F.S. et al. Goat milk oligosaccharides are antiinflammatory in rats with hapten-induced colitis. J Nutr. 2006; 136:672-6.

33. Vulevic J., Juric A., Tzortzis G., Gibson G.R. A mixture of trans-galactooligosaccharides reduces markers of metabolic syndrome and modulates the fecal microbiota and immune function of overweight adults. J Nutr. 2013; 143:324-31. doi:10.3945/ jn.112.166132.

34. Vulevic J., Drakoularakou A., Yaqoob P., Tzortzis G., Gibson G.R. Modulation of the fecal microflora profile and immune function by a novel trans-galactooligosaccharide mixture (B-GOS) in healthy elderly volunteers. Am J Clin Nutr. 2008; 88:1438-46.

35. Neish A.S. Microbes in gastrointestinal health and disease. Gastroenterology 2009;136:65-80

36. Barrett E.L., Kwan H.S. Bacterial reduction of trimethylamine oxide. Annu Rev Microbiol. 1985;39:131-149.

37. Smith J.L., Wishnok J.S., Deen W.M. Metabolism and excretion of methylamines in rats. Toxicol Appl Pharmacol. 1994; 125(2):296-308.

38. Zhang A.Q., Mitchell S.C., Smith R.L. Dietary precursors of trimethylamine in man: a pilot study. Food Chem Toxicol. 1999; 37(5):515-520.

39. Bain M.A., Fornasini G., Evans A.M. Trimethylamine: metabolic, pharmacokinetic and safety aspects. Curr Drug Metab. 2005; 6(3):227-240.

40. Bennett B.J. et al. Trimethylamine-N-oxide, a metabolite associated with atherosclerosis, exhibits complex genetic and dietary regulation. Cell Metab. 2013; 17(1):49-60.

41. Cashman J.R. et al. In vitro and in vivo inhibition of human flavin-containing monooxygenase form 3 (FMO3) in the presence of dietary indoles. Biochem Pharmacol. 1999; 58(6):1047-1055.

42. Tang W.H. et al. Intestinal microbial metabolism of phosphatidylcholine and cardiovascular risk. N Engl J Med. 2013; 368(17):1575-1584.

43. Miller C.A. et al. Effect of egg ingestion on trimethylamine-N-oxide production in humans: a randomized, controlled, dose-response study [published online ahead of print June 18, 2014]. Am J Clin Nutr. doi:ajcn.087692.

44. Koeth R.A. et al. Intestinal microbiota metabolism of L-carnitine, a nutrient in red meat, promotes atherosclerosis. Nat Med. 2013; 19(5):576-585.

45. Brown J.M., Hazen S.L. The Gut Microbial Endocrine Organ: Bacterially-Derived Signals Driving Cardiometabolic Diseases. Annual review of medicine. 2015; 66:343-359. doi:10.1146/annurevmed-060513-093205.

46. Rhee E.P. et al. A combined epidemiologic and metabolomic approach improves CKD prediction. J Am Soc Nephrol. 2013; 24(8):1330-1338.

47. Tang W.H. et al. Prognostic value of elevated levels of intestinal microbe-generated metabolite, trimethylamine-N-oxide, in patients with heart failure: refining the gut hypothesis. J Am Coll Cardiol. 2013;61(10_S). doi:10.1016/S0735-1097(13)60750-0.

48. Gao X., Liu X., Xu J., Xue C., Xue Y., Wang Y. Dietary trimethylamine $\mathrm{N}$-oxide exacerbates impaired glucose tolerance in mice fed a high fat diet. J Biosci Bioeng. 2014:S1389-1723(14)00081-4.

49. Hofmann A.F., Hagey L.R. Bile acid chemistry, biology, and therapeutics during the last 80 years: historical aspects. J Lipid Res. 2014 (In Press).

50. Watanabe M., Houten S.M., Mataki C. et al. Bile acids induce energy expenditure by promoting intracellular thyroid hormone activation. Nature. 2006; 439:484-9. [PubMed: 16400329]

51. Thomas C., Gioiello A., Noriega L. et al. TGR5-mediated bile acid sensing control glucose homeostasis. Cell Metab. 2009; 10:167-77. [PubMed: 19723493]

52. Pols T.W., Nomura M., Harach T. et al. TGR5 activation inhibits atherosclerosis by reducing macrophage inflammation and lipid loading. Cell Metab. 2011; 14:747-57. [PubMed: 22152303]

53. Ma K., Saha P.K., Chan L.L., Moore D.D. Farnesoid X receptor is essential for normal glucose homeostasis. J Clin Invest. 2006; 116:1102-9. [PubMed: 16557297] 
54. Downes M., Verdecia M.A., Roecker A.J. et al. A chemical, genetic, and structural analysis of the nuclear bile acid receptor FXR. Mol Cell. 2003; 11:1079-92. [PubMed: 12718892]

55. Sayin S.I., Wahlstrom A., Felin J. et al. Gut microbiota regulates bile acid metabolism by reducing the levels of tauro-beta-muricholic acid, a naturally occurring FXR antagonist. Cell Metab. 2013; 17:225-35. [PubMed: 23395169]

56. USDA. USDA Database for the Choline Content of Common Foods: Release 2 (2008). USDA Web site.http://www.ars.usda.gov/Services/ docs.htm?docid=6232. Updated May 17, 2013. Accessed on July 24, 2014.

57. Tang W.H. Wilson, Stanley L. Hazen. The contributory role of gut microbiota in cardiovascular disease. Journal of Clinical Investigation, 2014

58. Lekawanvijit S. et al. The uremic toxin adsorbent AST-120 abrogates cardiorenal injury following myocardial infarction. PLoS One. 2013; 8(12):e83687.

59. Yamamoto S. et al. Oral activated charcoal adsorbent (AST-120) ameliorates extent instability of atherosclerosis accelerated by kidney disease in apolipoprotein E-deficient mice. Nephrol Dial Transplant. 2011;26(8):2491-2497.

60. Ito S. et al. Reduction of indoxyl sulfate by AST-120 attenuates monocyte inflammation related to chronic kidney disease. J Leukoc Biol. 2013; 93(6):837-845.

61. Rebouche C.J., Seim H. Carnitine metabolism and its regulation in microorganisms and mammals. Annu Rev Nutr. 1998;18:39-61.8

62. Bain M.A., Faull R., Milne R.W., Evans A.M. Oral L-carnitine: metabolite formation and hemodialysis. Curr Drug Metab. 2006; 7(7):811-816.
63. Craciun S., Balskus E.P. Microbial conversion of choline to trimethylamine requires a glycyl radical enzyme. Proc Natl Acad Sci U S A. 2012; 109(52):21307-21312.

64. Craciun S., Marks J.A., Balskus E.P. Characterization of choline trimethylamine-lyase expands the chemistry of glycyl radical enzymes. ACS Chem Biol. 2014; 9(7):1408-1413.

65. Zhu Y. et al. Carnitine metabolism to trimethylamine by an unusual Rieske-type oxygenase from human microbiota. Proc Natl Acad Sci U S A. 2014; 111(11):4268-4273.

66. Delzenne N.M., Neyrinck A.M., Backhed F., Cani P.D. Targeting gut microbiota in obesity: effects of prebiotics and probiotics. Nat Rev Endocrinol. 2011; 7:639-46. [PubMed: 21826100]

67. Messenger J., Clark S., Massick S., Bechtel M. A review of trimethylaminuria: (fish odor syndrome). J Clin Aesthet Dermatol. 2013; 6(11):45-48.

68. Saha J.R., Butler V.P.Jr, Neu H.C. et al. Digoxin-inactivating bacteria: identification in human gut flora. Science. 1983; 220:325-327. [PubMed: 6836275]

69. Thomas C.M., Nielsen K.M. Mechanisms of, and barriers to, horizontal gene transfer between bacteria. Nat Rev Microbiol. 2005; 3:711-21. [PubMed: 16138099]

70. Hehemann J.H., Correc G., Barbeyron T. et al. Transfer of carbohydrate-active enzymes from marine bacteria to Japanese gut microbiota. Nature. 2010; 464:908-12. [PubMed: 20376150] 